\title{
TO STUDY THE SERUM LIPID PROFILE IN ISCHEMIC AND HEMORRHAGIC STROKE AMONG THE PATIENTS IN TERTIARY HEALTH CENTRE
}

\author{
ANKUR CHHARI ${ }^{1 *}$, KHANNA V N ${ }^{1}$, RAHUL JAIN $^{1}$, VISHNU PAL $^{2}$ \\ ${ }^{1}$ Department of Medicine, PCMS and RC, Bhopal, Madhya Pradesh, India. ${ }^{2}$ Department of Anatomy, PCMS and RC, Bhopal, Madhya Pradesh, \\ India. E-mail: dr.ankur85@gmail.com
}

Received: 01 December 2021, Revised and Accepted: 15 January 2021

\section{ABSTRACT}

Objectives: To assess, correlate and compare the levels of various parameters of lipid profile in patients with ischemic and hemorrhagic stroke.

Methods: The study was conducted as an observational study at the Department of Medicine, People's Hospital on 100 patients presenting with focal neurological deficit, altered sensorium, or with CT/MRI findings suggestive of stroke during the study duration of 18 months. Based upon the type of stroke, patients were categorized into two groups, i.e. ischemic stroke and hemorrhagic stroke. NCEP-ATP III guidelines were used for estimation of dyslipidemia and association of dyslipidemia was observed with type of stroke.

Results: Of 100 cases, ischemic stroke was documented in $74 \%$ cases, whereas $26 \%$ of patients presented with hemorrhagic stroke. Two groups were comparable in terms of baseline characteristics ( $p>0.05$ ). Mean total cholesterol levels and total cholesterol and total cholesterol to HDL ratio was significantly higher in ischemic stroke as compared to hemorrhagic stroke $(\mathrm{p}<0.05)$. However, mean serum HDL level was significantly lower in patients with ischemic stroke as compared to hemorrhagic stroke $(\mathrm{p}<0.05)$. Total cholesterol, and total cholesterol: HDL ratio showed statistically significantly negative correlation with type of stroke and positive correlation was noted between HDL and hemorrhagic stroke.

Conclusion: The prevalence of stroke is rising rapidly and the age of presentation of stroke is reducing. Ischemic stroke is the most common type of stroke whereas hemorrhagic stroke is less commonly observed in less than one-third of patients. Dyslipidemia is a significant risk factor for ischemic stroke. Raised Total cholesterol, and total cholesterol: HDL levels and lower HDL levels are independent predictors of ischemic stroke.

Keywords: Stroke, Dyslipidemia, Correlation, Total cholesterol: HDL ratio.

(C) 2022 The Authors. Published by Innovare Academic Sciences Pvt Ltd. This is an open access article under the CC BY license (http://creativecommons.org/ licenses/by/4.0/) DOI: http://dx.doi.org/10.22159/ajpcr.2022v15i2.43732. Journal homepage: https://innovareacademics.in/journals/index.php/ajpcr

\section{INTRODUCTION}

Stroke also called cerebrovascular accident or brain attack is characterized by neurological deficit which is due to acute focal injury to the central nervous system [1]. According to the Global stroke factsheet, new cases of stroke are reported in 13.7 million individuals each year. Every fourth individual over the age of 25 years is expected to experience a stroke in their lifetime. Incidence of stroke increase with age and more than $60 \%$ of strokes are reported in the age group of more than 70 years. Men and women are equally affected [2]. Various risk factors predispose to the development of stroke which includes increasing age, male gender, family history, ethnicity, genetic factors, lifestyle factors (such as smoking, alcohol), low socioeconomic status, diabetes, hypertension, heart disease, hypercholesterolemia, and obesity [3]. Atherosclerosis is considered as most common underlying pathology in stroke which leads to the formation of atherothrombotic plaque. Changes in lipid profile are considered as important risk factors in the occurrence of stroke. However, it has been documented that in patients with stroke, serum lipid levels are affected as a result of accompanying stress and increased production of catecholamines [4].

Deranged serum lipid levels in stroke have been associated with significant mortality. Literature suggests an inverse relationship between total cholesterol levels and mortality in patients with hemorrhagic stroke, whereas raised serum cholesterol has been documented in ischemic stroke [5]. It is important to assess the levels of lipids in patients with various types of stroke so as to appropriately guide the lipid-lowering therapy. The lipid-lowering drugs may help in reducing the morbidity as well as mortality associated with stroke by adapting primary and secondary preventive measures $[6,7]$. The present study was conducted at tertiary care center to assess, correlate and compare the levels of various parameters of lipid profile in patients with ischemic and hemorrhagic stroke.

\section{METHODS}

The study was conducted as an observational study at the Department of Medicine, People's College of Medical Sciences and People's Hospital on 100 patients presenting with focal neurological deficit, altered sensorium, and with CT/MRI findings suggestive of stroke during the study period of December 01, 2018 to May 31, 2020 (18 months). All the patients above 14 yrs across both the genders presenting with focal neurological deficit, altered sensorium, and CT/MRI findings consistent with ischemic or hemorrhagic stroke were included in the study whereas patients under the age of 14 years; on lipid-lowering agents; with a past history of stroke, stroke secondary to trauma, demyelination disorders, transient ischemic attack or patients with any neurological deficit due to meningitis and encephalitis and due to other infective etiologies were excluded from the study.

After obtaining ethical clearance from Institute's ethical committee, all the patients fulfilling inclusion criteria and giving written consent were enrolled. Detailed history regarding sociodemographic variables such as age, gender, socioeconomic status was obtained and entered in questionnaire. Detailed history regarding presenting complaints, duration of symptoms, presence of comorbidities, addiction, diet, and comorbid conditions were enquired and noted. Further, all the patients were subjected to detailed general and systemic examination including neurological examination. Venous samples were obtained under strict aseptic precautions and subjected to CBC, lipid profile, and blood glucose estimation using standard protocol. National Cholesterol Education Program-Adult Treatment Panel III (NCEPATP III) guidelines [8] were used for the estimation of dyslipidemia. Further, all the patients were subjected to neuroimaging study with either CT scan or MRI. 
The selected patients were divided into two categories based upon the type of stroke:

Group 1 - Ischemic stroke $(\mathrm{n}=74)$

Group 2 - Haemorrhagic stroke $(n=26)$.

\section{Statistical analysis}

Data were compiled using MS Excel and analyzed using IBM SPSS software version 20. Data were grouped and expressed as frequency and percentage whereas numerical data were expressed as mean and standard deviation. The two groups were compared with respect to confounding variables such as age, gender, and smoking. using Chisquare test. Chi-square test was also applied to assess the difference in proportions of various parameters between patients with ischemic and hemorrhagic stroke. Pearson correlation coefficient was used to assess the correlation between type of stroke and parameters of lipid profile. $P$ value $<0.05$ was considered statistically significant.

\section{RESULTS}

In the present study, out of 100 cases, ischemic stroke was documented in $74 \%$ of cases whereas $26 \%$ of patients presented with hemorrhagic stroke (Table 1)

The mean age of patients with ischemic stroke and hemorrhagic stroke was $60.08 \pm 13.8$ and $57.08 \pm 12.12$ years, respectively. Majority of patients with hemorrhagic stroke belonged to 61 to 70 years of age, whereas maximum patients with hemorrhagic stroke belonged to 51 to 60 years of age. About $73 \%$ of patients with ischemic stroke were males as compared to $61.5 \%$ males with hemorrhagic stroke. Tobacco addiction was noted in $63.5 \%$ of patients with ischemic stroke and $57.7 \%$ of patients with hemorrhagic stroke. Two groups were comparable in terms of baseline characteristics ( $p>0.05$ ) (Table 2)

Table 1: Distribution of patients according to baseline characteristic

\begin{tabular}{|c|c|c|c|c|c|}
\hline \multirow[t]{2}{*}{$\begin{array}{l}\text { Baseline } \\
\text { characteristic }\end{array}$} & \multicolumn{2}{|c|}{$\begin{array}{l}\text { Ischemic stroke } \\
(n=74)\end{array}$} & \multicolumn{2}{|c|}{$\begin{array}{l}\text { Hemorrhagic } \\
\text { stroke }(n=26)\end{array}$} & \multirow[t]{2}{*}{ p value } \\
\hline & $\mathbf{n}$ & $\%$ & $\mathbf{n}$ & $\%$ & \\
\hline \multicolumn{6}{|l|}{ Age group } \\
\hline$\leq 40$ & 8 & 10.8 & 2 & 7.7 & \multirow[t]{5}{*}{0.162} \\
\hline $41-50$ & 11 & 14.9 & 9 & 34.6 & \\
\hline $51-60$ & 18 & 24.3 & 7 & 26.9 & \\
\hline $61-70$ & 22 & 29.7 & 3 & 11.5 & \\
\hline$>70$ & 15 & 20.3 & 5 & 19.2 & \\
\hline \multicolumn{6}{|l|}{ Gender } \\
\hline Male & 54 & 73 & 16 & 61.5 & \multirow[t]{2}{*}{0.27} \\
\hline Female & 20 & 27 & 10 & 38.5 & \\
\hline \multicolumn{6}{|l|}{ Tobacco } \\
\hline Yes & 47 & 63.5 & 15 & 57.7 & \multirow[t]{2}{*}{0.59} \\
\hline No & 27 & 36.5 & 11 & 42.3 & \\
\hline \multicolumn{6}{|l|}{ Alcohol } \\
\hline Yes & 24 & 32.4 & 7 & 26.9 & \multirow[t]{2}{*}{0.60} \\
\hline No & 50 & 67.6 & 19 & 73.1 & \\
\hline
\end{tabular}

Table 2: Comparison of comorbidities between two groups

\begin{tabular}{|c|c|c|c|c|c|}
\hline \multirow[t]{2}{*}{ Comorbidities } & \multicolumn{2}{|c|}{$\begin{array}{l}\text { Ischemic stroke } \\
(n=74)\end{array}$} & \multicolumn{2}{|c|}{$\begin{array}{l}\text { Hemorrhagic } \\
\text { stroke }(n=26)\end{array}$} & \multirow[t]{2}{*}{$p$ value } \\
\hline & $\mathbf{n}$ & $\%$ & $\mathbf{n}$ & $\%$ & \\
\hline Diabetes & & & & & 0.162 \\
\hline Present & 34 & 45.9 & 4 & 15.4 & 0.006 \\
\hline Absent & 40 & 54.1 & 22 & 84.6 & \\
\hline \multicolumn{6}{|l|}{ Hypertension } \\
\hline Present & 30 & 40.5 & 17 & 65.4 & 0.04 \\
\hline Absent & 44 & 59.5 & 9 & 34.6 & \\
\hline \multicolumn{6}{|l|}{ CAD } \\
\hline Present & 8 & 10.8 & 3 & 11.5 & 0.92 \\
\hline Absent & 66 & 89.2 & 23 & 88.5 & \\
\hline
\end{tabular}

In the present study, diabetes was observed in significantly higher proportions of cases with ischemic stroke whereas hypertension was present in significantly higher proportions of patients with hemorrhagic stroke $(\mathrm{p}<0.05)$ (Table 3)

In the present study, borderline high and high cholesterol level was noted in significantly higher proportions of patients with ischemic stroke as compared to hemorrhagic stroke $(\mathrm{p}<0.05)$. Similarly, the Total cholesterol: HDL ratio was raised $(>6)$ in $29.7 \%$ of patients with ischemic stroke and $3.8 \%$ of patients with hemorrhagic stroke. Total cholesterol: HDL ratio was significantly raised in patients with ischemic stroke as compared to hemorrhagic stroke $(\mathrm{p}<0.01)$.

However, no such association between type of stroke and other lipid parameters was documented in the present study ( $p>0.05$ ) (Table 4).

In the present study, cholesterol ( $\mathrm{p}=0.038)$, and total cholesterol: HDL ratio $(p=0.001)$ showed statistically significant negative correlation with the type of stroke, i.e., total cholesterol, and total cholesterol: HDL ratio was higher in ischemic stroke and decreased significantly among patients with hemorrhagic stroke $(p<0.05)$. However, a statistically significant positive correlation was noted between HDL and hemorrhagic stroke $(\mathrm{p}=0.011)$.

\section{DISCUSSION}

Cholesterol has been thought to be differently involved in the pathogenesis of ischemic and hemorrhagic stroke [9]. The presentation aimed to determine the correlation of lipid levels with type of stroke. Hemorrhagic stroke mainly results from uncontrolled hypertension. Uncontrolled hypertension leads to lipohyalinosis and arteriolosclerosis which might be the underlying pathology of hemorrhagic stroke [10]. In the present study, hypertension was the most common comorbidity observed in $47 \%$ of cases of overall stroke, and hypertension was significantly associated with hemorrhagic stroke $(65.4 \%)$ as compared to ischemic stroke (40.5\%). Grace et al. [11] and Manornej et al. [12] also observed hypertension to be the most common risk factor for all strokes and hemorrhagic stroke, respectively.

Dyslipidemia, i.e., changes in lipid profile are independent risk factor associated with stroke. Deranged levels of lipid in stroke may result from stress and increased production of catecholamines [4]. Chronic dyslipidemia, particularly, raised cholesterol levels impair the function of the endothelium of blood vessels by producing superoxide and oxygen-free radicals. These changes increase the risk of atherosclerosis and most common content of atherosclerotic plaques is cholesterol. HDL cholesterol which is protective whereas LDL cholesterol is contributory to atherosclerosis. Observation of dyslipidemia in stroke is important as the use of lipid-lowering agents in these patients may reduce mortality and morbidity among patients with stroke $[6,7]$. Total cholesterol levels were deranged in significantly higher proportions of patients with ischemic stroke as compared to hemorrhagic stroke $(\mathrm{p}<0.05)$. Total cholesterol levels showed statistically significant negative correlation with hemorrhagic stroke $(p=0.038)$ in our study. These findings were supported by findings of Onwuegbuzie et al. in which dyslipidemia was observed in $35.8 \%$ of the patients and most among them, high TC was noted in $31 \%$ patients [13]. Togha et al. documented a statistically significant relationship between ischemic stroke and total cholesterol similar to the present study [14].

HDL levels more than 40 are considered to be protective for atherogenesis and stroke. In the present study, we documented a statistically significant positive correlation between HDL and hemorrhagic stroke $(\mathrm{p}=0.011)$. Similarly, Shrivastav et al. documented significantly lower levels of HDL in patients with hemorrhagic stroke as compared to healthy controls. HDL-C showed positively non-significantly association with hemorrhagic stroke $(\mathrm{p}=0.3785)[15]$ 
Table 3: Association of type of stroke with dyslipidemia

\begin{tabular}{|c|c|c|c|c|c|c|}
\hline \multirow[t]{2}{*}{ Lipid profile } & \multicolumn{2}{|c|}{ Ischemic stroke $(n=74)$} & \multicolumn{2}{|c|}{ Hemorrhagic stroke $(n=26)$} & \multirow[t]{2}{*}{$\chi^{2}$} & \multirow[t]{2}{*}{ p value } \\
\hline & $\mathbf{n}$ & $\%$ & $\mathbf{n}$ & $\%$ & & \\
\hline \multicolumn{7}{|l|}{ Total cholesterol (mg/dl) } \\
\hline Borderline high (200-239) & 19 & 25.7 & 3 & 11.5 & & \\
\hline High $(\geq 240)$ & 12 & 16.2 & 1 & 3.8 & & \\
\hline \multicolumn{7}{|l|}{$\mathrm{LDL}(\mathrm{mg} / \mathrm{dl})$} \\
\hline Optimal $(<100)$ & 29 & 39.2 & 13 & 50 & 3.04 & 0.55 \\
\hline Near optimal (100-129) & 19 & 25.7 & 4 & 15.4 & & \\
\hline Borderline High (130-159) & 18 & 24.3 & 6 & 23.1 & & \\
\hline High (160-189) & 5 & 6.8 & 3 & 11.5 & & \\
\hline Very high $(\geq 190)$ & 3 & 4.1 & 0 & 0 & & \\
\hline \multicolumn{7}{|l|}{ Triglycerides (mg./dl) } \\
\hline Normal $(<150)$ & 52 & 70.3 & 18 & 69.2 & 0.70 & 0.70 \\
\hline Borderline (150-199) & 10 & 13.5 & 5 & 19.2 & & \\
\hline High (200-499) & 12 & 16.2 & 3 & 11.5 & & \\
\hline \multicolumn{7}{|l|}{$\mathrm{HDL}(\mathrm{mg} / \mathrm{dl})$} \\
\hline Normal (40-60) & 21 & 28.4 & 10 & 38.5 & & \\
\hline High $(>60)$ & 5 & 6.8 & 4 & 15.4 & & \\
\hline \multicolumn{7}{|l|}{ Total cholesterol: HDL ratio } \\
\hline$<4$ & 21 & 28.4 & 16 & 61.5 & 11.58 & 0.003 \\
\hline $4-6$ & 31 & 41.9 & 9 & 34.6 & & \\
\hline$>6$ & 22 & 29.7 & 1 & 3.8 & & \\
\hline
\end{tabular}

Table 4: Correlation between lipid profile and type of stroke

\begin{tabular}{lll}
\hline Lipid profile & Pearson correlation coefficient & Sig. \\
\hline Total cholesterol & -0.208 & 0.038 \\
LDL & -0.202 & 0.06 \\
Triglycerides & -0.053 & 0.603 \\
HDL & 0.253 & 0.011 \\
TC: HDL & -0.359 & 0.001 \\
\hline
\end{tabular}

Total: HDL cholesterol ratio is indicator with high predictive value for stroke and coronary artery disease as compared to isolated parameters [16]. In the present study, mean total cholesterol: HDL ratio showed a significant negative correlation with ischemic stroke ( $\mathrm{p}=0.001$ ). Liu et al. concluded that TC/HDL-C ratio are better predictor of ischemic stroke among men whereas TG are better predictor for stroke in males [16]. The findings of our study were supported by findings of Zhang et al. in which high total/HDL cholesterol ratio were significantly associated ischemic stroke in both males and females as compared to hemorrhagic stroke [17]. Overall, total cholesterol: HDL cholesterol followed by HDL and total cholesterol were significantly correlated with ischemic stroke in the present study, whereas LDL and triglyceride levels showed similar distribution in both ischemic and hemorrhagic stroke.

\section{CONCLUSION}

The prevalence of stroke is rising rapidly and the age of presentation of stroke is reducing. Ischemic stroke is a most common type of stroke whereas hemorrhagic stroke is less common observed in less than onethird patients. Stroke was observed in higher proportions of males. Diabetes was associated with ischemic stroke whereas hypertension was significant risk factor for hemorrhagic stroke. Dyslipidemia is a significant risk factor for ischemic stroke. Among various parameters of lipid profile, raised total cholesterol, and total cholesterol: HDL levels and lower HDL levels were independent predictors of ischemic stroke. The study observed no significant association of type of stroke with LDL and triglyceride levels.

\section{AUTHORS' CONTRIBUTIONS}

All the authors have contributed to the preparation and editing of this research article.

\section{CONFLICT OF INTEREST}

None.

AUTHOR'S FUNDING

None.

\section{REFERENCES}

1. Sacco RL, Kasner SE, Broderick JP, Caplan LR, Connors JJ, Culebras A, et al. An updated definition of stroke for the $21^{\text {st }}$ century: A statement for healthcare professionals from the American Heart Association/American Stroke Association. Stroke 2013;44:2064-89.

2. World Stroke Organization. WSO Global Stroke Fact Sheet. World Stroke Organization; 2020. Available from https://www.world-stroke. org/assets/downloads/WSO Global_Stroke Fact Sheet.pdf [Last accessed on 2020 Oct 24]

3. Arboix A, Alió J. Cardioembolic stroke: Clinical features, specific cardiac disorders and prognosis. Curr Cardiol Rev 2010;6:150-61.

4. Adibhatla RM, Hatcher JF. Altered lipid metabolism in brain injury and disorders. Subcell Biochem 2008;49:241-68.

5. Austin MA, King MC, Vranizan KM, Krauss RM. Atherogenic lipoprotein phenotype. A proposed genetic marker for coronary heart disease risk. Circulation 1990;82:495-506.

6. Kirshner HS. Medical prevention of stroke. South Med J 2003;96:354-8.

7. Bhalla A, Gupta OP, Gupta SB. Predicting mortality in stroke. Neurol India 2002;50:279-81.

8. Eckel RH, Cornier MA. Update on the NCEP ATP-III emerging cardiometabolic risk factors. BMC Med 2014;12:115.

9. Prospective Studies Collaboration. Cholesterol, diastolic blood pressure, and stroke: 13000 strokes in 450000 people in 45 prospective cohorts. Lancet 1995;346:1647-53.

10. Grace MN, Shameer VK, Rajesh KR, Raghavan R, Sreejesh S. A prospective observational study on the clinical profile of ischemic stroke in a tertiary care centre in Thrissur, Kerala. Int J Med Res Rev 2016;4:1371-5.

11. Manorenj S, Siddiqui IA, Muralikrishna P, Anand K, Sagari N. Is low cholesterol a predisposing factor for primary intracerebral hemorrhage? A South Indian perspective. J Clin Diagn Res 2018;12:5.

12. Onwuegbuzie GA, Rifkatu SR. Serum lipid profile of patients with ischemic stroke in a tertiary centre in Nigeria. IOSR J Dent Med Sci 2016;15:60-4

13. Togha M, Gheini MR, Ahmadi B, Khashaiar P, Razeghi S. Lipid profile in cerebrovascular accidents. Iran J Neurol 2011;10:1

14. Shrivastav D, Singh AN, Kushwaha JS, Tripathi P, Verma MK, 
Saxena N. Diagnostic value of lipid profile in hemorrhagic stroke patients. Era J Med Res 2019;6:1-6.

15. Millán J, Pintó X, Muñoz A, Zúñiga M, Rubiés-Prat J, Pallardo LF, et al. Lipoprotein ratios: Physiological significance and clinical usefulness in cardiovascular prevention. Vasc Health Risk Manag 2009;5:757.
16. Liu X, Yan L, Xue F. The associations of lipids and lipid ratios with stroke: A prospective cohort study. J Clin Hypertens 2019;21:127-35.

17. Zhang Y, Tuomilehto J, Jousilahti P, Wang Y, Antikainen R, Hu G. Total and high-density lipoprotein cholesterol and stroke risk. Stroke 2012;43:1768-74. 